\title{
Sharp Initial Disagreements Then Consensus in a Student Led Whole-Class Discussion
}

\author{
Brant E. Hinrichs \\ Department of Physics, Drury University, 729 N. Drury Lane, Springfield, MO 65802
}

\begin{abstract}
After learning Newton's 2nd Law, students in a university modeling-based introductory physics class are asked to imagine a box sliding across a floor and slowing to a stop. Although they've had extensive experience with friction in the context of energy, this is their first exposure to friction within the context of force. They are asked to make different representations for this scenario, including a system schema, and force diagram. During their small group work, students quickly run into a difficulty: there are only two interactions with the box (contact, gravitational), so there should only be two forces, yet the box is slowing, which means it must have unbalanced forces in the direction of acceleration. In this paper, preliminary evidence from a student-led whole-class discussion is presented showing how the group reasons through sharp disagreement in their initial ideas to come to a useful consensus.
\end{abstract}

Keywords: system schema, discourse management, board meeting, whole class discussion, socio-physics norms PACS: $01.40 . F k, 01.40 . \mathrm{gb}, 01.50 . \mathrm{F}-$

\section{INTRODUCTION}

This paper is a preliminary case study illustrating how students in a university modeling classroom $[1,2,3,4]$, taught by an experienced teacher, overcome multiple disagreements in their initial ideas to reach a useful whole-class consensus on a problem coordinating multiple representations [4]. This research is useful because no one has previously reported that a physics classroom is able to perform this kind of challenging work on its own without the teacher as leader.

\section{CLASSROOM CONTEXT}

The context of this case study is one section of an introductory calculus-based physics course taught using University Modeling Instruction pedagogy and taken by all science majors at Drury University. Twenty-six students were randomly divided into six groups. This is their fourth different group so far. It's near the end of the semester. Content-wise, the class had studied kinematics, energy [5], and Newton's Laws. They've had extensive experience making and using many different representations: graphs, motion diagrams, system schema [6,7], 1-d force diagrams, etc.

Format-wise, the class uses Modeling Discourse Management [3], a learning-community approach that explicitly focuses on the epistemology of science. It's designed to help students understand that science is tentative and evolving and that knowledge and meaning are constructed and shared through dialogue. In Modeling Discourse Management (MDM), an instructor poses a problem and students work in their small groups to create a solution on a 24 "x32" whiteboard. They then form a large circle with their whiteboards held to face the rest of the class (Fig. 1) and conduct a semi-structured discussion ("board meeting") to reach a consensus on the best solution to the given problem.

By this time in the semester students are very at ease in their small groups, and relatively at ease in the large group. They run the entire process themselves the instructor is outside the circle and not involved in the discussion. This suggests that the instructor's efforts in using MDM have led to a productive modeling-specific sociophysics norm [8]: students are obligated to achieve a consensus where all representations are consistent with each other and make sense with the problem statement. Sociophysics norms differ from general social norms in that they concern the normative aspects of classroom actions that are specifically physics related [9], for example, what counts as

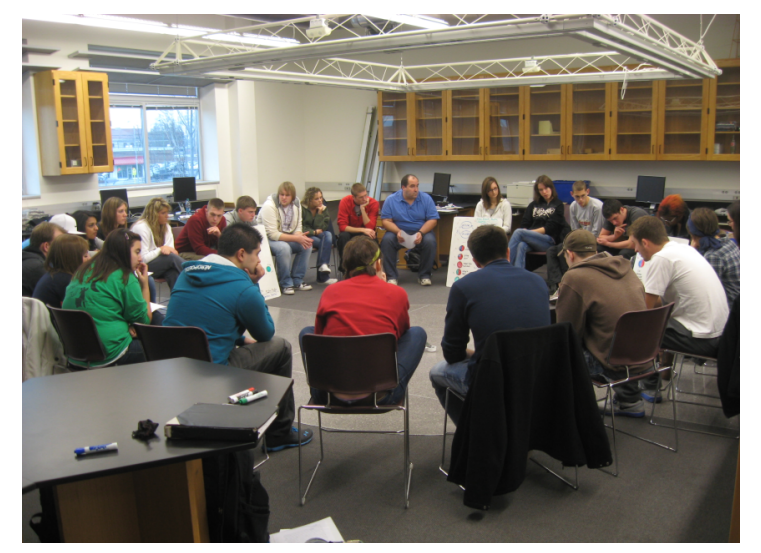

FIGURE 1. Large circle group with whiteboards facing in. Instructor sits outside the circle to the right (off camera). 
a useful system schema or force diagram.

\section{PHYSICS PROBLEM GIVEN}

Fig. 2 shows the specific problem students worked for this case study. We will focus only on questions $\# 1$ and \#4 since that is what the whole-class discussion centered on. By this time in the course students knew how to add arbitrarily angled vectors, that there are the same number of forces as interactions, and that acceleration and force total must point in the same direction.

The small groups worked for 20 minutes to make their whiteboards. Only group \#3 asked for significant assistance. The instructor gave them strong hints [10] which helped them make a force diagram consistent with their schema and graphs.

\section{CLASSROOM DATA}

There are three sources of data for this case study: photos, audio, and field notes. An audio recording of the large group discussion was made and transcribed. Despite difficulties in hearing different students in the recording, the author is fairly confident of identifying who spoke and what he or she said. In addition, photos of each small group's whiteboard were taken. Lastly, the author took daily field notes throughout the semester. These notes were used to inform the entire data analysis process.

\section{Small Group Whiteboard Evidence}

Fig. 3 shows answers from small group white-boards to question \#1 (system schema). Groups \#1 and \#2 had nearly identical boards so only group \#1's is shown. The other four groups had nearly identical schema, so only group \#3's is shown. The class eventually chose

\footnotetext{
You walk into a room and see a book sliding across the floor and slowing down. The book then comes to rest. You did not see what caused the book to start moving in the first place and do not care.

1) Make a system schema for this situation.

2) Define an appropriate coordinate system and make graphs of velocity and acceleration.

3) What would a graph of Force Total look like? Sketch it.

4) Now using your system schema from one, make a force diagram for the box. Remember you can only have the same number of forces as interactions!

5) What is the hard part about doing question four? Explain.

6) How would you write Newton's second law for this situation? Try.

7) Put your ideas and conclusions on a whiteboard.
}

FIGURE 2. Problem statement given to students for this case study (@ 2003 Dwain Desbien, used with permission).
Fig. $3 \mathrm{~b}$ as their consensus. The key point to note is the sharp disagreement between the initial ideas the groups brought to the whole class discussion.

A system schema shows relevant objects, the interactions between them, and a choice of system [6, 7]. Thus far in the course, two types of interactions have been identified - contact, "c", (between two objects that physically touch), and gravitational, "g", (between Earth and anything else). All groups appropriately have two objects (book and floor/Earth) but disagree about the number of interactions. Fig. 3a suggests that groups \#1 and \#2 thought of friction as a new type of interaction, "f", so they have three interactions between book and floor/earth. In contrast, the other four groups (Fig. 3b) have decided that there are just the usual two types of interactions. This is one of the sharp disagreements between initial group ideas.

Figs. 4 and 5 show answers from small group whiteboards to question \#4 (force diagram). All groups have appropriately applied the rule that there must be the same number of forces in their force diagram as there are interactions in their system schema (three for groups \#1 and \#2 because their system of the book is experiencing three interactions and two for groups \#3-\#6). Of the four groups that did not have friction as a new interaction, groups \#3 and \#5 decided that friction is just a part of the contact interaction (Figs. 4b, 5), while the other two groups did not specifically indicate friction in their force diagrams (Figs. 4c, 4d). The class eventually chose Fig. 5 as their consensus.

Again, note the disagreement between the initial ideas of the groups. Two groups (Fig. 4a) have three forces while four groups (Fig. 4b-d) have two forces. Even in the latter case, they don't all agree - there are three different force diagrams with just two forces. These are the other sharp disagreements between initial group ideas.

\section{Large Group Discussion Evidence}

The point of highlighting differences between initial student answers is not to belittle or demean. Rather, in the instructor's experience, whole class discussions that have such differences often fail to come to consensus on their own, without the teachers'

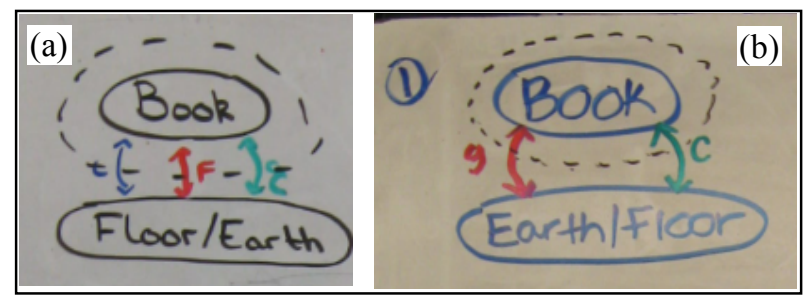

FIGURE 3. System Schema of groups: (a) \#1, \#2, (b) \#3, \#4, \#5, \#6. 


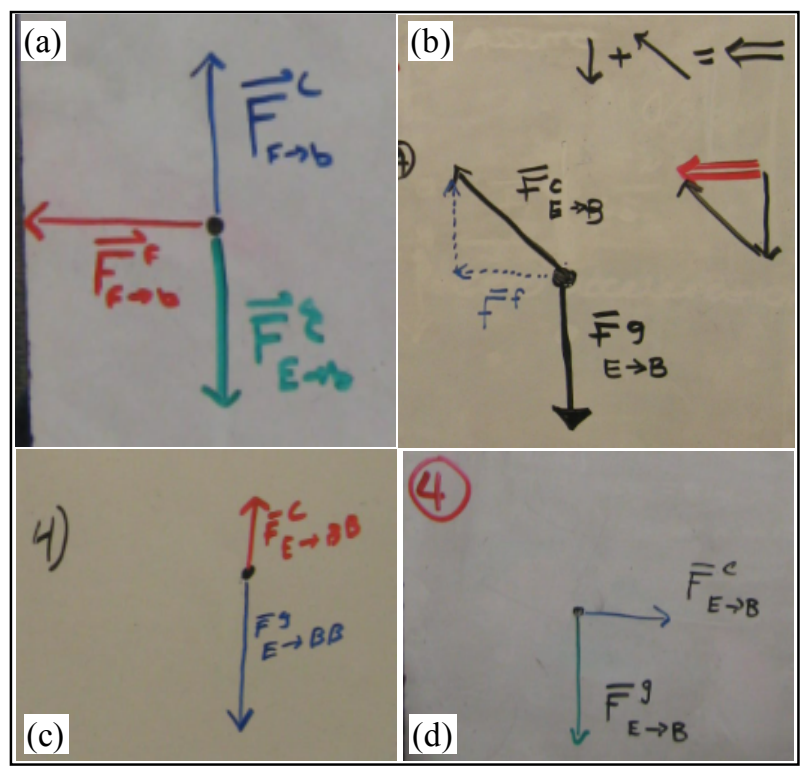

FIGURE 4. Force diagrams of groups (a) \#1, \#2, (b) \#3, (c) \#4 and (d) \#6.

intervention because: (i) students aren't able to identify and articulate the key differences in the answers they have, and/or (ii) they aren't able to find a principle or path of reasoning that helps them select one answer as better than the other(s). The aim of this section is to show that in this particular discussion the class was still able to come to a useful consensus on their own because they did achieve (i) and (ii).

The Modeling Instructor's goal of large group discussion is that students notice the important discrepancies between their answers and work together toward a consensus that resolves them. In this case study, groups must coordinate their system schema and force diagram representations with their motion graphs. This coordination hinges on whether friction is an interaction in the schema or not. If it is, then Fig. $4 \mathrm{a}$ is appropriate, otherwise Fig. 5 is. This section highlights parts of the whole-class discussion to illustrate how the group achieved a resolution and consensus.

First some background details. The entire discussion was 16 minutes. Group \#1 started, and for the first $7.5 \mathrm{~min}$., each group in turn presented their whiteboard. As group \#1 started there was immediate consensus on the graphs, so thereafter all groups focused almost exclusively on presenting and explaining their group's force diagram.

Group \#4 was the only group to get a significant amount of Q\&A as they presented. Students from groups \#1, \#5, and \#6 pointed out that the force total for group \#4's force diagram pointed down rather than left as acceleration did. A member of group \#4 acknowledged they had been confused about it and were not sure. The class never revisited this issue.

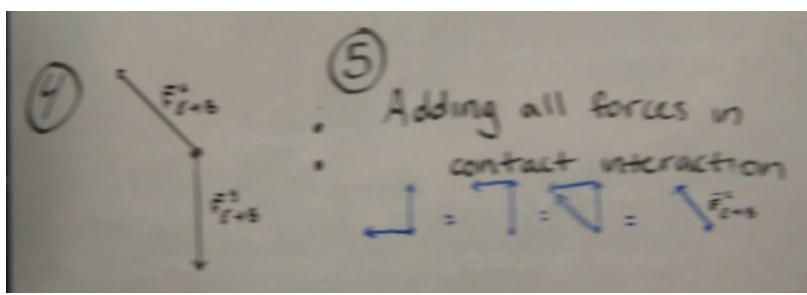

FIGURE 5. Group \#5's (blurry) force diagram. The large group discussed this extensively. Labels on the left diagram are $\vec{F}_{E \rightarrow B}^{c}$ for the tilted top vector and $\vec{F}_{E \rightarrow B}^{g}$ for the bottom.

Group \#6 admitted they were stumped by the force diagram and hoped the class would teach them. After their turn, there was a seamless transition into a wideranging, continuously productive whole-class discussion aimed at finding consensus. Fourteen (of 26) students made substantive contributions to the conversation. All but group \#4 had at least two members contribute. The instructor only spoke twicetrivially once near the start, and once near the end to refocus the conversation after a detour into air resistance. In what follows, all names are pseudonyms, and a number next to a name indicates the group. For example [Sam,1] means Sam is in group \#1.

At least two members each of groups \#1 and \#2 clearly stated they thought of friction as a new type of interaction. They also explicitly discussed what this implied for the schema. For example:

[Sam,1] 01:25 We were asked to begin. And...we set up a schema that had a third interaction in it, it had friction in it, Um.

[Andy,2] 11:30 Don't we have to account for that (friction) in our schema somehow?...I think you have to

In fact, Kim wondered about the angled vector in group \#5's force diagram, which was constructed from a vertical and horizontal (friction) vector:

[Kim, 1] 09:17 I just wanna know why you can... say there's a vector for friction if it's not an interaction within your schema.

She led an extensive back and forth about this point for almost a minute.

After the $7.5 \mathrm{~min}$ mark, three students gradually helped focus the discussion by identifying key differences amongst the boards that needed to be resolved:

[Min,2] 08:28 So essentially...you have to figure out whether or not there is a third interaction, which is friction, whether it's its own interaction, or whether it's a part of the contact interaction. [Sam,1] 09:36 So, basically, the major difference between ... our group consensuses are...centered around whether or not friction is an interaction and if friction isn't an interaction then it has to be 
a part of the contact

[Ralph,5] 11:59 Well... it basically comes down to is friction an interaction by itself or is friction part of the contact interaction already there?

Then Karen chimed in, with a very careful parsing of language:

[Karen,6] 12:18 I have a problem saying friction is an interaction though because friction is defined as.. a.., like, caused by a contact, like caused by an interaction, so like how can friction be an interaction if it's caused by another interaction

The class was excited by this and many tried to speak. Here are just a few:

[Stan,1] 12:38 Isn't friction, like, due to contact, it's a product of it so is it, is it possible to label it as its own interaction or is it due to the contact? [Jim,4] 12:52 It's part of the contact interaction. [Min,2] 13:05 because...it it, I mean it's created by the contact

The only sustained pause in the $16 \mathrm{~min}$ then occurred at 13:33. After ten seconds of silence, Jim spoke:

[Jim,4] 13:43 I think that friction is inherent to contact, so we need to have it as con, part of the contact interaction.

Finally, Stan came up with two questions that seemed really help many in the class:

[Stan,1] 14:09 I think it's good idea to look at it, as as whether or not it's an interaction, can you have a friction interaction without a contact interaction?

[many] No

[Stan,1] Sooo, can you have friction as its own interaction?

[many] No

After a diversion into a question about air resistance, the class came to a final consensus:

[Stan, 1] 15:27 Sooo, how do we label...friction, with, on the contact interaction, on the system schema. That's the next question. If we're agreeing that friction can't be another interaction. It's due to, it's a product of the contact.

[Sue, 6] I think we'll leave it out of the schema and put it in the assumptions.

[Jim, 4] I like the way, I like the way they (group \#5) did it where they just showed the two different types of contact interaction, um, and then showed the resulting vector, as the contact interaction, so I thought that was good.

[Min, 2] Yeah, [Sam, 1] Nice, [Stan, 1] Alright [others] Laughs of satisfaction

\section{DISCUSSION}

Student-led whole-class discussions can sometimes fail to find consensus for the two reasons (i) \& (ii) stated earlier. But here, the class was clearly able to overcome both potential difficulties. Multiple students identified the problem as figuring out if friction was a third type of interaction or not. While Karen began the path of reasoning to help the class decide, Jim nurtured it, and Stan's last two questions cemented it.

This paper is about describing what happened in a productive class rather than explaining why it happened or how to achieve it. The author is actively compiling additional examples of such productive whole-class discussions from other contexts in an effort to generalize and address those later two questions. Meanwhile, here are two possible reasons the author thinks this particular whole-class discussion was productive.

First, there were very different answers with very different assumptions. The groups saw the clear differences, wanted to resolve them, and enough individuals had sufficient understanding of the relevant physics that they could.

Second, students were very comfortable asking questions or making statements repeatedly or being wrong in front of their peers. The establishment through MDM of a sociophysics norm of reaching consensus via identifying and resolving key physics differences had overcome typical student reluctance to take risks or question each other's thinking and they were eager to negotiate meaning, which is just the type of scientific reasoning that is needed in physics classrooms.

\section{ACKNOWLEDGMENTS}

Thanks to Miki, Eads, \& Fenn; Dwain Desbien for a sabbatical spent shadowing his Modeling Discourse; Ellie Sayer for fruitful conversation; Colleen Megowan \& Andy Johnson for very extensive comments.

\section{REFERENCES}

1. D. Hestenes, Am. J. Phys. 55, 440-454 (1987).

2. M. Wells, D. Hestenes, and G. Swackhamer, Am. J. Phys. 63, 606-619 (1995).

3. D. M. Desbien, D.M., Ph.D. Thesis, ASU, 2002.

4. E. Brewe, Am. J. Phys. 76 (12), 1155-1160 (2008).

5. E. Brew, Phys. Rev. Spec. Topics, PER 7, 020106 (2011).

6. L. Turner, Phys.Teach. 41, 404-408 (2003).

7. B. E. Hinrichs, AIP Conf. Proc. 790, 117 (2005).

8. A. Johnson, PhD Thesis, San Diego State (1999).

9. K. McClain, P Cobb, J. Res. Math Ed. 32 (3) (2001).

10. J. Durden, talk, AAPT Winter Meeting (2011). 